\title{
THE APPLICATION OF SHIFT METHOD USING MOVING LASER ON CANCER CASES IN HEAD AREA USING 3DCRT AND VMAT TECHNOLOGY
}

\author{
Samsun*, Nursama Heru Apriantoro, Rudi Kurniawan, Agnes Dwika Septiti \\ Department of Radiodiagnostic and Radiotherapy Engineering, Health Polytechnic of Jakarta II, \\ Jl. Hang Jebat III Block F3 Jakarta Selatan 12720
}

*Corresponding Author Email: aiman_052005@yahoo.com

Received: 29 June 2019

Revised: 28 December 2019

Accepted: 30 December 2019

Published: 31 December 2019

SPEKTRA: Jurnal Fisika dan Aplikasinya

p-ISSN: 2541-3384

e-ISSN: 2541-3392

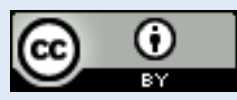

\begin{abstract}
The accuracy of patient position is essential to achieve the purpose of radiotherapy. Therefore, before radiotherapy, the isocenter of the patient must be determined to find out the target location. Verification serves to determine the isocenter that shifts from the reference point on the CT simulator. This research was conducted using field observation method, comparing the results of the isocenter after verification with the isocenter on the moving laser. Eight (8) samples from primary data were taken. Shift data was obtained from the average value and standard deviation. The instrument used was a linear accelerator of clinic iX type and On-Board Imager. The results obtained were the average lateral coordinate point of $0.055 \mathrm{~cm}$; at the longitudinal isocenter point of $0.10 \mathrm{~cm}$; and at the vertical isocenter point of $0.053 \mathrm{~cm}$. It was concluded that the average value o the isocenter shift was less than $0.3 \mathrm{~cm}$. This method can be used because the shift does not exceed the tolerance limit.
\end{abstract}

Keywords: moving laser, verification, on-board imager 


\section{INTRODUCTION}

Radiotherapy is a treatment using ionizing radiation sourced from radioactive energy. It is one of the medical treatment that plays a role in cancer treatment using ionizing radiation. Ionizing radiation can be in the form of magnetic waves, X-rays and gamma rays or from groups of alpha, beta, and neutron particles. A considerable amount of cancer patients who go to hospitals receive radiotherapy. The radiation received is not just single radiotherapy but it can also be combined with chemotherapy or surgery [1]. Generally, a cancer patient receives more than one type of radiation. Radiotherapy also called irradiation, X-ray therapy, or the popular term "dibestral" aims to destroy cancer tissue [2]. At least it works to reduce the size of cancer tissue or eliminate the symptoms and disorders accompanying it. Sometimes, it is even used for prevention (prophylactic).

The success of radiotherapy requires treatment in multidisciplinary science, including Radiation Oncologists, medical physicists, dosimetrists, radiation therapists, radiographers, electromedical technicians, and nurses. In the flow of radiotherapy patients, a medical decision determines whether the patient needs radiotherapy or not. The patient may also get a combination of surgery, chemotherapy, and radiotherapy.

Radiotherapy is one of the modalities for cancer treatment [1]. Radiotherapy uses the ionizing radiation to kill cancer cells and maintain healthy tissue around cancer [2]. Nowadays, radiotherapy techniques such as IMRT and VMAT have been worked well in increasing the dose to the local focus area of tumors with a high degree of accuracy [3].

Before conducting the radiation process, the patient must be verified [3]. Verification serves to determine the irradiation reference point that shifts from the reference point on the simulator [4]. This is usually performed in the treatment room by the radiation therapist. Verification might be possible for some patients, but it might also be difficult if seeing the data from the World Health Organization (WHO) and the International Cancer Control Union (UICC) that predict an increase in the number of cancer patients each year [2].

\section{METHOD}

\section{Shift Method Using Moving Laser}

This research used the experimental data collection method by conducting experiments using moving laser as a new method for the shifting from reference points to irradiation points. The instruments used in this research were laser moving application of LAP isomark 3.1, a worksheet for recording Treatment Planning data on CT Simulator, 2D/2D Match application, and Print Out while the verification used On-Board Imager (OBI). The researchers conducted data processing and analysis through observation. Then, the results of the $\mathrm{x}, \mathrm{y}$, and $\mathrm{z}$ coordinate shifts were obtained after verification. The collected data were processed statistically to determine the average shift that occurred in 8 samples. The results of data processing were then calculated using averages on the sample. 


\section{COUNTING EXPERIMENTS}

\section{Analysis using 3 DCRT and VMAT Technology}

This OBI provides three different forms of imaging models; 2-D radiographic acquisition, fluoroscopic image acquisition, and 3-D Cone Beam Computed Tomography (CBCT). There are two models for 2D digital radiography; standard and high quality. 2D radiograph obtained from OBI is called kilovoltage $(\mathrm{kV})$, while the image obtained from linac is called megavoltage (MV).

The 2D match application used two images obtained when the patient was in the radiotherapy treatment room and two reference images of Digital Reconstructed Radiographs (DRR) or simulator images. Digital Reconstructed Radiograph (DRR) was obtained from CT Planning image. To equalize, the two sets of images must be perpendicular to each other. Generally, the image used was the projection of AP (Anteroposterior) and Lateral.

AP (Anteroposterior) view was used to determine the lateral and longitudinal table shift, while the lateral view was to find out the vertical and longitudinal table shift. This could only be obtained by using the On-Board Imager (OBI) system. A green graticule scale showed the original treatment planning, while the red graticule scale showed the results from OBI. The two graticules originally coincided. Reference image or Digital Reconstructed Radiograph (DRR) would shift in the direction corresponding to the structure of the image obtained from the On-Board Imager (OBI).

The results obtained were in the form of longitudinal, vertical, and lateral isocenter point shift values using a moving laser after verification with OBI (On-Board Imager). In the shift data, there were negative values (-) and positive values (+). These values only indicated the shift direction. The research results were described in the form of a shift table as described in TABLE 1 .

TABLE 1. The shift of Moving Laser Isocenter and OBI Verification

\begin{tabular}{|c|c|c|c|c|c|c|}
\hline \multirow{3}{*}{$\begin{array}{l}\text { Location on the } \\
\text { patient head }\end{array}$} & \multicolumn{6}{|l|}{ Shift } \\
\hline & \multicolumn{3}{|c|}{ moving laser with conversion value on linac $(\mathrm{cm})$} & \multicolumn{3}{|c|}{ Verification with OBI (cm) } \\
\hline & Lat & Long & Vert & Lat & Long & Ver \\
\hline Point A & 0 & 103.2 & 14.0 & 0 & 103.19 & 14.0 \\
\hline Point B & 999.0 & 107.6 & 13.2 & 999.0 & 107.7 & 13.19 \\
\hline Point C & 0 & 104.8 & 17.5 & 0 & 104.9 & 17.5 \\
\hline Point D & 999.3 & 103.5 & 12.7 & 999.5 & 103.4 & 12.71 \\
\hline Point E & 2.0 & 102.9 & 15.4 & 2.1 & 103.1 & 15.42 \\
\hline Point F & 999.8 & 102.8 & 14.5 & 999.78 & 102.7 & 14.5 \\
\hline Point G & 999.13 & 102.6 & 14.1 & 999.1 & 102.8 & 14.0 \\
\hline Point $\mathrm{H}$ & 999.9 & 100.7 & 16.1 & 0.1 & 100.9 & 16.0 \\
\hline
\end{tabular}


Positive (+) and negative (-) values in each sample (attached) worked as directions for the shift of radiation therapy table. Therefore in calculating the average, it was considered absent.

TABLE 2. Average Results of Moving Laser Shift

\begin{tabular}{lll}
\hline Coordinate point & $\mathrm{N}$ & Average $(\mathrm{cm})$ \\
\hline Lateral & 8 & 0.0676 \\
Longitudinal & 8 & 0.1015 \\
Vertical & 8 & 0.0537 \\
\hline
\end{tabular}

In TABLE 2, it was obtained an average at lateral coordinate $0.067 \mathrm{~cm}$; at the longitudinal isocenter point $0.10 \mathrm{~cm}$; and at the vertical isocenter point $\mathrm{b} 0.053 \mathrm{~cm}$; this showed that the average value at the three isocenter points did not exceed the tolerance limit of $0.3 \mathrm{~cm}$.

The table showed that of the average results at the lateral coordinate point $0.067 \mathrm{~cm}$; at the longitudinal isocenter point $0.10 \mathrm{~cm}$; and at the vertical isocenter point $0.053 \mathrm{~cm}$; this showed that the average value at the three isocenter points did not exceed the tolerance limit of $0.3 \mathrm{~cm}$. After verification, the value of the isocenter area shift in patients with cancer in the head region using a moving laser was caused by the movement made by the patient from the CT simulator room to the LINAC room and the laser difference used in the CT simulator room and treatment room. There was a possibility of human error in the implementation of treatments, such as patient set-up by RTT, miscalculation of shifts by medical physicists, and uncooperative patients.

Determination of the tolerance limit was performed to ensure the accuracy of the target area location so that the distribution of the dose to be given did not deviate from the target location that had been contoured by the radiation oncologist. Therefore, it is crucial to notice the accuracy because if there was a shift of more than $0.03 \mathrm{~cm}$, the distribution of absorbed doses to be received would be greater to the area of organs at risk around and the distribution of absorbed doses to be received by the target organ.

\section{RESULT AND DISCUSSION}

Evaluation of patient shift data conducted on the moving laser was performed after the patient was in simulation by determining the isocenter. So, a reference point or lateral longitudinal and vertical coordinate point was obtained in which the point would be used to calculate the actual shift of the target to be irradiated. Then, the simulation patient data were sent to the TPS via aria software.

To conduct the verification on Linac, first clicked 2D/2D (2D/2D up mode) on the monitor. Second, clicked download axis, then clicked atomically. Third, pressed the OBI motion to enable and auto buttons simultaneously to release the detector and OBI cube from the parking position. Then, pressed the expose button (it should not be released until the scanning process was completed) until the anatomical image of the entire pelvic anterior-posterior position was 
seen. Then, pressed the OBI motion to enable and retract the button to return the position of the detector and OBI cube in the parking position. Click the Lateral for lateral view. Turned the gantry to $90^{\circ}$, then press the OBI motion enable and auto button simultaneously to release the detector and OBI cube from the parking position. Then, press the exposure button (should not be released until the scanning process is complete) until the anatomical image of the entire pelvic lateral position is seen. Turn the gantry direction to $0^{0}$, then pressed the OBI motion to enable and auto button simultaneously to release the detector and OBI cube from the parking position. Clicked analyze, then adjusted the verification image with the results of the CT scan during the simulation by shifting the pelvic image in the box to match the background image (the results of the CT scan). Large shift can be seen in the shift column on the monitor. Next, click done and clicked Apply. Data from the verification shift would be recorded in the patient's medical record even though the verification shift data was stored on ARIA software.

The data sent to the TPS was then processed by medical physicists to treat patients, one of which was to calculate the shift from the reference point. After the shift data was obtained, the medical physicist sent the shift data to the Dorado LAP application. Marked the shift point with a blue marker to be an isocenter during the treatment. However, before the treatment was performed, RTT must verify to check the accuracy of isocenter first, in order to achieve the Radiotherapy purpose.

From the research results, the use of OBI verification helped to reduce the radiation doses in critical organs.

The use of eye-shielding as a radiation protection measure should be performed to reduce the dose received by the eye from the verification process. The use of eye-shielding could only be performed while using OBI, not in tumor/cancer irradiation. Eye shielding could not be applied during verification using Cone-Beam CT (CBCT), because it might cause artifacts that interfere with the image.

\section{CONCLUSION}

The average value at the lateral coordinate point was $0.067 \mathrm{~cm}$; at the longitudinal isocenter point was $0.10 \mathrm{~cm}$, and at the vertical isocenter point was $0.053 \mathrm{~cm}$. Moving the laser method can be used to determine the SSD isocenter because the accuracy of determining the isocenter does not exceed the tolerance limit of $0.3 \mathrm{~cm}$.

The cause of the shift in the isocenter is the patient movement from the CT simulator room to the moving laser room.

\section{REFERENCES}

[1] Fauchon, F., Hasselblatt, M., Jouvet, A., Champier, J., Popovic, M., Kirollos, R., ... Vasiljevic, A. (2013). Role of surgery, radiotherapy and chemotherapy in papillary tumors of the pineal region: A multicenter study. Journal of Neuro-Oncology, 112(2), 223-231. https://doi.org/10.1007/s11060-013-1050-5 
[2] Gantchew, M. (2010). Radiotherapy risk profile. Rentgenologiya I Radiologiya, 49(4), 282-285.

[3] Verhey LJ. Immobilizing and positioning patients for radiotherapy. Semin Radiat Oncol. 2013;5(2):100-14.

[4] Djordjevic, Milos. (2007) Evaluation of Geometric Accuracy and Image Quality of an On-Board Imager (OBI). Stockholm University and Karolinska Institute 